\title{
Erratum to: Surgical Weight Loss: Impact on Energy Expenditure
}

\section{David Thivel • Katrina Brakonieki • Pascale Duche • Béatrice Morio • Yves Boirie • Blandine Laferrère}

Published online: 16 February 2013

(C) Springer Science+Business Media New York 2013

\section{Erratum to: OBES SURG \\ DOI 10.1007/s11695-012-0839-1}

In the original publication, an erroneous date is given in the information on Prior Presentations in the Acknowledgements section. The correct date for the AHRQ Health Information Technology conference is June 2-4, 2010 as opposed to September 27, 2010.

In the original publication, the given and surnames of the fourth and fifth authors were reversed. The correct names are Béatrice Morio (B. Morio) and Yves Boirie (Y. Boirie).

The online version of the original article can be found at http://dx.doi.org/ 10.1007/s11695-012-0839-1.

D. Thivel $\cdot$ K. Brakonieki $\cdot$ B. Laferrère

New York Obesity Nutrition Research Center,

Department of Medicine, St. Luke's Roosevelt Hospital Center,

New York, NY 10025, USA

D. Thivel $(\bowtie) \cdot$ P. Duche

Laboratory of the Metabolic Adaptations to Exercise under

Physiological and Pathological Conditions (AME2P),

EA 3533, Clermont University, Blaise Pascal University,

BP 80026, 63171 Aubière cedex, France

e-mail: thiveldavid@hotmail.com

\section{K. Brakonieki $\cdot$ B. Laferrère}

Division of Endocrinology and Diabetes, Department of Medicine,

St. Luke's Roosevelt Hospital Center, New York, NY 10025, USA

\section{B. Laferrère}

Columbia University College of Physicians \& Surgeons,

New York, NY, USA
B. Morio $\cdot$ Y. Boirie

Unité de Nutrition Humaine, Clermont Université,

Université d'Auvergne, BP 10448,

63000 Clermont-Ferrand, France

B. Morio · Y. Boirie

INRA, UMR 1019, UNH, CRNH Auvergne,

63000 Clermont-Ferrand, France

\section{Y. Boirie}

Clinical Nutrition Department, CHU Clermont-Ferrand, Clermont-Ferrand 63003, France 